International Mathematical Forum, 1, 2006, no. 2, 51-60

\title{
SOME GENERAL NOTIONS OF STOCHASTIC ORDERINGS FOR WEIGHTED RELIABILITY AND UNCERTAINTY MEASURES WITH APPLICATIONS
}

\author{
Broderick O. Oluyede \\ Department of Mathematical Sciences \\ Georgia Southern University, Statesboro, GA 30460, USA \\ Boluyede@GeorgiaSouthern.edu
}

\begin{abstract}
In this note, stochastic comparisons of reliability measures and related functions are presented. Inequalities for uncertainty of a residual life distribution and certain modified cross-entropy or discrimination information measures under weighted models are established. Comparisons of the expected uncertainty about the remaining lifetime of a component for weighted conditional distributions and unweighted conditional distributions are presented.
\end{abstract}

Mathematics Subject Classification: 62N05, 62B10

Keywords: Stochastic inequalities, Weighted distribution functions, Integrable function.

\section{Introduction}

Weighted distributions in general and length-biased distributions in particular, are of tremendous practical importance in a wide variety of areas in probability and statistics. Length-biased sampling is widely used for the collection and analysis of wildlife data (Patil and Rao, 1978), fiber data (Daniels, 1942) or lifetime data (Zelen and Feinleib, 1969, Gupta and Keating, 1985). A variety of methods have been developed for the estimation of the survival function, density function and other related functions under length-biased sampling. See Vardi 1982, 1985; Bhattacharyya et al, 1988; and references therein. 
This paper is concerned with the establishment of stochastic inequalities and bounds for reliability and uncertainty measures when the underlying models are weighted and length-biased. Let $\mathrm{X}$ be a non-negative random variable with distribution function $F$, survival function $\bar{F}$ and probability density function(pdf) $f$. The weighted random variable $X_{W}$ has a survival function given by

$$
\bar{G}_{W}(x)=\bar{F}(x)\left(W(x)+T_{F}(x)\right) / E_{F}(W(X)),
$$

where $T_{F}(x)=\int_{x}^{\infty}\left(\bar{F}(u) W^{\prime}(u) d u\right) / \bar{F}(x)$, and $W^{\prime}(u)=d W(u) / d u$, assuming that $W(x) \bar{F}(x) \rightarrow 0$ as $x \rightarrow \infty$. The corresponding probability density function (pdf) of the weighted random variable $X_{W}$ is given by

$$
g_{W}(x)=W(x) f(x) / \delta^{*},
$$

where $\delta^{*}$ is a normalizing constant. Note that $0<\delta^{*}=E_{F}(W(X))<\infty$. Patil and Rao (1978) referred to (2) as a weighted distribution with weight function $W(x) \geq 0$. The purpose of this note is to obtain inequalities and compare reliability and uncertainty measures for weighted distributions. Section 2 is concerned with some basic definitions and utility notions. In section 3 , stochastic comparisons and orderings involving reliability functions are presented. Section 4 is concerned with inequalities for uncertainty measures in weighted distributions. Results for modified cross-entropy or discrimination information measures are presented. It is shown that the expected uncertainty contained in the conditional distribution of $X_{W}-t$ given $X_{W}>t$, about the remaining lifetime of the component for monotone weight functions is larger than the expected uncertainty in the conditional distribution of $X-t$ given $X>t$, about the remaining lifetime of the component for sampling under the unweighted distribution. In section 5, some applications and examples are presented.

\section{Some Definitions and Utility Notions}

In this section, I present some definitions and useful notions. Let $\mathcal{F}$ be the set of absolutely continuous distribution functions satisfying

$$
F(0)=0, \lim _{x \rightarrow \infty} F(x)=1, \sup \{x: F(x)<1\}=\infty .
$$

Note that if the mean of a random variable with distribution function in $\mathcal{F}$ is finite, it is positive. Let $\bar{F}(x)$ and $\bar{G}(x)$ be the survival functions of the random variables $X$ and $Y$ respectively.

Definition 2.1 If $X$ and $Y$ are in $\mathcal{F}$, then $X$ is said to be larger than $Y$ in (i) stochastic ordering $\left(X \geq{ }_{\text {st }} Y\right)$ if $\bar{F}(x) \geq \bar{G}(x)$ for all $x \geq 0$; (ii) D ordering 
$\left(X \geq_{D} Y\right)$ if

$$
\limsup _{x \rightarrow \infty} \frac{\bar{F}(x)}{\bar{G}(x)}<\infty,
$$

or equivalently, if $\bar{F}(x) \leq k \bar{G}(x)$ for all $x \geq 0$, and some $k \geq 1$.

The following definition is due to Basu and Ebrahimi [1]. The authors studied and developed analytical properties for this class of life distributions.

Definition 2.2 A life distribution $F$ is $k$-harmonic new better than used in expectation ( $k-H N B U E)$ if

$$
\left(\mu^{k} / t\right) \int_{0}^{t} \delta_{F}^{-k}(x) d x \geq 1
$$

for all $t>0$, where $k \geq 1, \mu_{F}=\int_{0}^{\infty} \bar{F}(y) d y$, and $\delta_{F}(x)=\int_{x}^{\infty} \bar{F}(y) d y / \bar{F}(x)$ is the mean residual life function of a distribution function $F$. The inequality is reversed for k-harmonic new worst than used in expectation (k-HNWUE).

In the next definition, we give an ordering of the life distributions $F$ and $G$. This definition is used in section 3 to compare and order the distribution function $F$ and the weighted or sampling distribution function $G_{W}$.

Definition 2.3 Let $F$ and $G$ be life distribution functions with mean residual life functions $\delta_{F}$ and $\delta_{G}$ respectively. We say $F$ is $k$-harmonic mean residual life average dominated by $G$ if

$$
\left\{t^{-1} \int_{0}^{t} \delta_{F}^{-k}(x) d x\right\}^{-1} \geq\left\{t^{-1} \int_{0}^{t} \delta_{G}^{-k}(x) d x\right\}^{-1}
$$

for all $t>0$ and $k \geq 1, \delta_{F}(x)=\int_{x}^{\infty} \bar{F}(y) d y / \bar{F}(x)$ is the mean residual life function of a distribution function $F$. The inequality is reversed for $G$-harmonic mean residual life average ( $k$-HMRLA) dominated by $F$.

Definition 2.4 A random variable $X$ with distribution function $F$ is said to be decreasing hazard rate (DHR) if and only if $\bar{F}(x+t) / \bar{F}(x)$ is increasing in $x \geq 0$, for every $t \geq 0$.

\section{Some Comparisons and Orderings of Relia- bility Measures}

Let $f_{W}$ and $g_{W}$ be two non-negative functions, possibly weighted probability density functions that are integrable with respect to a $\sigma$-finite measure $\eta$. A natural and common approach to tail ordering of distribution functions $F$ and $G$ with probability density functions (pdf) $f$ and $g$ respectively is concerned 
with the rate at which the density tends to zero at infinity. A pdf $f$ is said to have a lighter tail than a pdf $g$ if $f(x) / g(x) \longrightarrow 0$ as $x \rightarrow \infty$. Let $g_{l}$ and $g_{W}$ be the length-biased and weighted probability density functions respectively. The length-biased probability density function is a weighted probability density function with weight function $W(x)=x$. The corresponding length-biased reliability function is given by

$$
\bar{G}_{l}(x)=\bar{F}(x) V_{F}(x) / \mu_{F},
$$

where $V_{F}(x)=E(X \mid X>x)$ is the vitality function. Note that $f(x) / g_{l}(x)=$ $\mu_{F} / x \longrightarrow 0$ as $x \rightarrow \infty$. That is, the length-biased distribution has heavier tail than the original distribution. Indeed

$$
\bar{G}_{l}(x) \geq \bar{F}(x)
$$

for all $x \geq 0$.

Theorem 3.1 Let $g_{W}(x)=W(x) f(x) / \delta^{*}$ be a weighted pdf with increasing weight function $W(x) \geq 0$. Then $\bar{G}_{W}(x) \geq \bar{F}(x)$ and

$$
\limsup _{x \rightarrow \infty} \frac{\bar{F}(x)}{\bar{G}_{W}(x)}<\infty,
$$

where $\bar{G}_{W}(x)=\left(\delta^{*}\right)^{-1} \int_{x}^{\infty} W(y) f(y) d y, 0<\delta^{*}=E(W(X))<\infty$.

Proof: Let $W(x)$ be increasing in $x \geq 0$. Then

$$
\bar{G}_{W}(x)=\frac{\bar{F}(x) E[W(X) \mid X>x]}{E(W(X))} \geq \bar{F}(x)
$$

for all $x \geq 0$, that is $\bar{G}_{W}(x)$ and $\bar{F}(x)$ are stochastically ordered. This implies that for some $k \geq 1$,

$$
k \bar{G}_{W}(x) \geq \bar{F}(x)
$$

for all $x \geq 0$.

Equivalently,

$$
\limsup _{x \rightarrow \infty} \frac{\bar{F}(x)}{\bar{G}_{W}(x)}<\infty,
$$

for all $x \geq 0$.

Theorem 3.2 Let $G_{W}$ be a weighted distribution function with increasing weight function $W(x), x \geq 0$. If $F$ is $k$-HNWUE then

$$
\left\{t^{-1} \int_{0}^{t} \delta_{F}^{-k}(x) d x\right\}^{-1} \geq \mu^{-k}
$$

and $G_{W}$ is $k$-HMRLA dominated by $F$ for $t>0$ and $k \geq 1$. 
Proof: Let $W(x)$ be increasing, then $\lambda_{G_{W}}(x) \leq \lambda_{F}(x)$ and $\delta_{G_{W}}(x) \geq \delta_{F}(x)$ for all $x \geq 0$, where $\lambda_{F}(x)=f(x) / \bar{F}(x)$. Clearly, $\delta_{G_{W}}^{-k}(x) \leq \delta_{F}^{-k}(x)$ for all $x \geq 0$ and $k \geq 1$, so that

$$
\left\{t^{-1} \int_{0}^{t} \delta_{G_{W}}^{-k}(x) d x\right\}^{-1} \geq\left\{t^{-1} \int_{0}^{t} \delta_{F}^{-k}(x) d x\right\}^{-1} \geq \mu^{-k},
$$

for $t>0$ and $k \geq 1$. Consequently, $G_{W}$ is $k$-HMRLA dominated by $F$.

Theorem 3.3 Let $G_{W}$ be a weighted distribution function with increasing weight function $W(x), x \geq 0$. If $G_{W}$ is $k$-HMRLA dominated by $F$, then $G_{W}$ is $(k+1)-H M R L A$ dominated by $F$ for $0<t \leq 1$ and $k \geq 1$.

Proof: Let $W(x)$ be increasing in $x$. For $0<t \leq 1$, we have

$$
\begin{aligned}
t^{-1} \int_{0}^{t} \delta_{F}^{-(k+1)}(x) d x & =t^{-1} \int_{0}^{t}\left\{\delta_{F}^{-k}(x)\right\}^{(k+1) / k} d x \\
& \geq t^{-1}\left\{\int_{0}^{t} \delta_{F}^{-k}(x) d x\right\}^{(k+1) / k}(1 / t)^{1 / k} \\
& =\left\{t^{-1} \int_{0}^{t} \delta_{F}^{-k}(x) d x\right\}^{(k+1) / k} \\
& \geq\left\{t^{-1} \int_{0}^{t} \delta_{G_{W}}^{-k}(x) d x\right\}^{(k+1) / k} \\
& \geq t^{-1} \int_{0}^{t} \delta_{G_{W}}^{-(k+1)}(x) d x
\end{aligned}
$$

The first inequality is due to Holder's inequality. The second inequality follow from the fact that $W(x)$ is increasing in $x$, so that $\delta_{G_{W}}(x) \geq \delta_{F}(x)$ for all $x \geq 0$, and $0<t \leq 1$. The third inequality is trivial.

Consequently,

$$
\left\{t^{-1} \int_{0}^{t} \delta_{G_{W}}^{-(k+1)}(x) d x\right\}^{-1} \geq\left\{t^{-1} \int_{0}^{t} \delta_{F}^{-(k+1)}(x) d x\right\}^{-1}
$$

\section{Comparison for Uncertainty Measures}

In this section, stochastic comparisons and inequalities for uncertainty and reliability measures are established. Consider two probability spaces $\left(\Omega, \Phi, \nu_{1}\right)$ and $\left(\Omega, \Phi, \nu_{2}\right)$ such that the probability measures $\nu_{1}$ and $\nu_{2}$ are absolutely continuous with respect to $\eta$. Let $\eta$ be a probability measure defined on $\Phi$ and equivalent to $\nu_{1}$ and $\nu_{2}$. Suppose $f_{1}(x)$ and $f_{2}(x)$ are Radon-Nikodym derivatives of $\nu_{1}$ and $\nu_{2}$ with respect to $\eta$. The basic uncertainty for a distribution function $F$ is the differential commonly referred to as the Shannon information measure [8] given by

$$
I(f)=-\int_{0}^{\infty} f(x) \log (f(x)) d x .
$$


The cross-entropy (Guiasu[5]) is

$$
I\left(f_{1}, f_{2}\right)=\int_{\Omega} \log \left(f_{1}(x) / f_{2}(x)\right) f_{1}(x) d \eta(x) .
$$

We define cross-entropy or discrimination information for residual life distribution of a component as

$$
I^{*}\left(f_{1}, f_{2} ; t\right)=\int_{t}^{\infty} f_{1}(x) \log \left(f_{1}(x) / f_{2}(x)\right) d x
$$

and

$$
I\left(f_{1}, f_{2} ; t\right)=\int_{t}^{\infty} \frac{f_{1}(x)}{\overline{F_{1}}(t)} \log \left(\frac{\left(f_{1}(x) / \overline{F_{1}}(t)\right)}{\left(f_{2}(x) / \overline{F_{2}}(t)\right)}\right) d x .
$$

The uncertainty of a residual life distribution of a component is given by

$$
\begin{aligned}
I(f ; t) & =-\int_{t}^{\infty}(f(x) / \bar{F}(t)) \log (f(x) / \bar{F}(t)) d x \\
& =-(\bar{F}(t))^{-1} \int_{t}^{\infty} f(x) \log (f(x) / \bar{F}(t)) d x .
\end{aligned}
$$

$I(f ; t)$ can be rewritten as

$$
I(f ; t)=\log (\bar{F}(t))-(\bar{F}(t))^{-1} \int_{t}^{\infty} f(x) \log (f(x)) d x .
$$

The definition given by (19) is due to Ebrahimi and Pellerey [4]. I( $f ; t)$ measures the expected uncertainty about the predictability of the remaining lifetime of the component in the conditional density of $X-t$ given $X>t$.

Theorem 4.1 Let $f_{W}$ and $g_{W}$ be two weighted probability density functions with weight function $W(x) \geq 0$. If $g_{W}(x) \geq f_{W}(x)$ for all $x \geq 0$, then

$$
D_{1}\left(f_{W}, g_{W} ; t\right) \geq I^{*}\left(f_{W}, g_{W} ; t\right) \quad \text { for all } t \geq 0,
$$

where $D_{1}\left(f_{W}, g_{W} ; t\right)=\int_{t}^{\infty}\left|g_{W}(x)-f_{W}(x)\right| d x$ and $I^{*}\left(f_{W}, g_{W} ; t\right)$ is given by $(17)$.

Proof: Note that

$$
\begin{aligned}
D_{1}\left(f_{W}, g_{W} ; t\right) & =\int_{t}^{\infty}\left|g_{W}(x)-f_{W}(x)\right| d x \\
& =-\int_{t}^{\infty}\left(f_{W}(x)-g_{W}(x)\right) d x \\
& =-\int_{t}^{\infty} f_{W}(x)\left(1-g_{W}(x) / f_{W}(x)\right) d x \\
& \geq-\int_{t}^{\infty} f_{W}(x)\left(\log \left(g_{W}(x) / f_{W}(x)\right)\right) d x
\end{aligned}
$$

where the last inequality follows from $\ln (y) \geq 1-(1 / y)$ for $y \geq 0$.

Similarly,

$$
D_{1}\left(f_{W}, g_{W} ; t\right) \geq I^{*}\left(g_{W}, f_{W} ; t\right) \text {, for all } t \geq 0 \text {. }
$$


Theorem 4.2 Let $g_{W}(x)=W(x) f(x) / E(W(X)), 0<E(W(X))<\infty$. If $W(x)$ is increasing, and $\bar{F}$ or $\bar{G}_{W}$ are DHR, then

$$
I\left(g_{W} ; t\right) \geq I(f ; t), \quad \text { for all } t \geq 0,
$$

where $I(f ; t)$ is given by (19).

Proof: Let $W(x)$ be increasing in $x$, then $g_{W}(x) / f(x)=W(x) / E(W(X))$ is an increasing function of $x$. Using the fact that $\bar{F}$ or $\bar{G}_{W}$ are DHR, we have

$$
g_{W}(x) / \bar{G}_{W}(x) \leq f(x) / \bar{F}(x)
$$

for all $x \geq 0$ and

$$
\bar{G}_{W}(x) \geq \bar{F}(x)
$$

for all $x \geq 0$. It follows that

$$
\frac{\bar{G}_{W}(t)}{\bar{F}(t)} \geq \frac{\int_{t}^{\infty} g_{W}(x) \log \left(g_{W}(x) / \bar{G}_{W}(t)\right) d x}{\int_{t}^{\infty} f(x) \log (f(x) / \bar{F}(t)) d x}
$$

and

$$
I\left(g_{W} ; t\right) \geq I(f ; t), \quad \text { for all } t \geq 0 .
$$

The last theorem compares the expected uncertainty contained in the conditional distribution of $X_{W}-t$ given $X_{W}>t$, about the remaining lifetime of the component when sampling is done unknowingly from the weighted distribution as opposed to the unweighted distribution.

Theorem 4.3 Let $X_{W}$ and $Y_{W}$ be two weighted random variables with distribution functions $F_{W}$ and $G_{W}$ respectively. If $\bar{F}_{W}$ or $\bar{G}_{W}$ are DHR then

$$
I\left(f_{W}, g_{W} ; t\right) \geq I^{*}\left(f_{W}, g_{W} ; t\right)
$$

for all $t \geq 0$, where $I\left(f_{W}, g_{W} ; t\right)$ and $I^{*}\left(f_{W}, g_{W} ; t\right)$ are given above.

Proof: Note that

$$
\begin{aligned}
I\left(f_{W}, g_{W} ; t\right) & =\int_{t}^{\infty} \frac{f_{W}(x)}{\bar{F}_{W}(t)} \log \left(\frac{f_{W}(x) / \bar{F}_{W}(t)}{\left(g_{W}(x) / \bar{G}_{W}(t)\right)}\right) d x \\
& \geq\left(\bar{F}_{W}(t)\right)^{-1} \int_{t}^{\infty} f_{W}(x) \log \left(\frac{f_{W}(x)}{g_{W}(x)}\right) d x
\end{aligned}
$$

The last inequality follows from the assumption of DHR which implies stochastic order between $F_{W}$ and $G_{W}$, so that $\log \left(\bar{G}_{W}(t) / \bar{F}_{W}(t)\right) \geq 0$, for all $t \geq 0$.

Consequently,

$$
I\left(f_{W}, g_{W} ; t\right) \geq I^{*}\left(f_{W}, g_{W} ; t\right) \quad \text { for all } t \geq 0 .
$$


Theorem 4.4 . Let $g_{W_{i}}(x)=\frac{W_{i}(x) f(x)}{E\left(W_{i}(X)\right)}$, and $0 \leq \alpha_{i}=E\left(W_{i}(X)\right) \leq \infty$, $i=1,2$. If $\frac{W_{2}(x)}{W_{1}(x)}$ is increasing in $x$, and $G_{W_{1}}$ or $G_{W_{2}}$ are DHR then

$$
I\left(g_{W_{2}} ; t\right) \geq I\left(g_{W_{1}} ; t\right)
$$

for all $t \geq 0$.

Proof: Let $K(x)=W_{2}(x) / W_{1}(x)$, then $K(x)=\alpha_{2} g_{W_{2}}(x) / \alpha_{1} g_{W_{1}}(x)$ is increasing in $x$ and $\bar{G}_{W_{2}}(x) \leq \bar{G}_{W_{1}}(x)$ for all $x \geq 0$.

Note that $K(x)$ increasing in $x$ and the assumption that $G_{W_{1}}$ or $G_{W_{2}}$ are DHR implies

$$
g_{W_{2}}(x) / \bar{G}_{W_{2}}(x) \leq g_{W_{1}}(x) / \bar{G}_{W_{1}}(x)
$$

for all $x \geq 0$.

We get

$$
\frac{\bar{G}_{W_{2}}(t)}{\bar{G}_{W_{1}}(t)} \geq \frac{\int_{t}^{\infty} g_{W_{2}}(x) \log \left(g_{W_{2}}(x) / \bar{G}_{W_{2}}(t)\right) d x}{\int_{t}^{\infty} g_{W_{1}}(x) \log \left(g_{W_{1}}(x) / \bar{G}_{W_{1}}(t)\right) d x}
$$

for all $x \geq 0$.

Consequently,

$$
I\left(g_{W_{2}} ; t\right) \geq I\left(g_{W_{1}} ; t\right)
$$

for all $t \geq 0$.

\section{Applications}

Let $F$ and $G_{W}$ be the original and weighted distribution functions respectively. We give some examples of the results presented in earlier sections.

1. Rayleigh Distribution. The Rayleigh pdf is given by

$$
f(x ; \beta)=2 \beta^{-1} x e^{-\beta x^{2}}, \quad \text { if } x>0 \text { and } \beta>0 .
$$

The corresponding length-biased pdf is given by

$$
g_{W}(x ; \beta)=4 \pi^{-1 / 2} \beta^{3 / 2} x^{2} e^{-\beta x^{2}}, \quad \text { if } x>0 \text { and } \beta>0 .
$$

Note that for $\beta \geq 1$, we have

$$
I\left(g_{W} ; t\right) \geq I(f ; t)
$$

for all $t \geq 0$. 
2. Exponential Distribution. The exponential distribution is one of the most useful model in reliability and survival analysis. The pdf is given by

$$
f(x ; \theta)=(1 / \theta) e^{-x / \theta}, \quad \text { if } x>0 \text { and } \theta>0 .
$$

The weighted reliability function with weight function $W(x)=x$ is given by

$$
\left.\bar{G}_{W}(x ; \theta)=(x+\theta) / \theta\right\} e^{-x / \theta}, \quad \text { if } x>0 \text { and } \theta>0 .
$$

The uncertainty of the residual lifetime for the length-biased distribution given by reduces to

$$
I\left(g_{W} ; t\right)=1+\log \left(\delta_{F}(t)\right)=1+\log (\theta) .
$$

Clearly, $I\left(g_{W} ; t\right) \geq I(f ; t)$, for all $t \geq 0$.

ACKNOWLEDGEMENTS. The author is grateful to the editor and the referee for taking the time to review this paper.

\section{References}

[1] A. P. Basu, and N. Ebrahimi, On k-Order Harmonic New Better Than Used in Expectation Distributions, Annals of the Institute of Statistical Mathematics, 36 (1) (1984), 87-100.

[2] B. B. Bhattacharyya, L. A. Franklin, and D. G. Richardson, A Comparison of Nonparametric Unweighted Length-Biased Density Estimation of Fibers, Communications in Statistics, A 17 (1988), 3629-3644.

[3] H. E. Daniels, A New Technique for the Analysis of Fiber Length Distribution in Wool, Journal of Textile Institute, 33 (1942), 137-150.

[4] N. Ebrahimi, and F. Pellerey, Partial Ordering of Survival Functions Based on the Notion of Uncertainty, Journal of Applied Probability, 32 (1995), 202-211.

[5] S. Guiasu, Information Theory with Applications, McGraw Hill, New York, 1977.

[6] R. C. Gupta, and J. P. Keating, Relations for Reliability Measures Under Length-Biased Sampling, Scandinavian Journal of Statistics, 13 (1985), 49-56.

[7] G. P. Patil, and C. R. Rao, Weighted Distributions and Size-Biased Sampling with Applications to Wildlife and Human Families, Biometrics, 34 (1978), 179-189. 
[8] C. E. Shannon, A Mathematical Theory of Communication, Bell System Technical Journal, 27 (1948), 379-423.

[9] Y. Vardi, Nonparametric Estimation in the Presence of Length Bias, Annals of Statistics, 10 (1969), 616-620.

[10] Y. Vardi, Empirical Distributions in Selection Bias Models, Annals of Statistics, 13 (1985), 178-203.

[11] M. Zelen, and M. Feinleib, On the Theory of Screening for Chronic Diseases, Biometrika, 56 (1969), 601-614.

Received: April 6, 2005 